\title{
Classifying emotion regulation strategies
}

\section{Candace M. Raio, Elizabeth V. Goldfarb, Karolina M. Lempert and Peter Sokol-Hessner}

In their recent article (The neural bases of emotion regulation. Nat. Rev. Neurosci. 16, 693-700 $(2015))^{1}$, Etkin, Büchel and Gross proposed testing emotion regulation using computational approaches that are anchored in reinforcement learning. Their framework classifies emotion regulation strategies as model-based (MB; computationally sophisticated, prospective processes that consider environmental structure) or model-free (MF; computationally efficient, reflexive processes relying on past reinforcement) ${ }^{2}$. Although linking emotion regulation to computational decision making is valuable, here we argue that the MB versus MF classification may be inadequate to capture the complexity of emotion regulation.

The authors use extinction as a key example of a MF regulation strategy. During extinction, conditioned responses diminish as a conditioned stimulus (CS) is no longer reinforced by an unconditioned stimulus (US). Extinction could be perceived as a MF strategy because it uses prediction error signals that emerge from recent reinforcement (that is, a CS no longer predicts an US). However, a standard observation is that after extinction learning, conditioned responses tend to re-emerge over time (a process known as spontaneous recovery) $)^{3,4}$. As MF reinforcement learning theories describe extinction as the process of 'unlearning' the CS-US association ${ }^{5}$, they cannot account for spontaneous recovery. Rather, extinction learning across species has been shown to have $\mathrm{MB}$ features $^{6,7}$. Specifically, a MB perception of the 'state' in which extinction learning occurs predicts the magnitude of spontaneous recovery. Further, modelling conditioned responses in humans using a MF learning strategy alone fits the data more poorly than a Bayesian 'latent cause' model that incorporates MB features ${ }^{7}$. Thus, extinction appears to reflect a complex interaction of MF learning with MB state representations ${ }^{8}$. Since even simple extinction learning cannot be described as purely $\mathrm{MF}$, it is unlikely that a MB-MF distinction will usefully distinguish between emotion regulation strategies ${ }^{9}$.

The limitations of the MB-MF distinction are further highlighted by recent work on decision making demonstrating the predictive power of hybrid MB-MF models and identifying complex relationships between these strategies. Such conceptualizations can include hierarchical organization of goals and sub-goals, in which MB-MF processes exist within each other (for example, a goal is selected in a MF sense, but implemented using $\mathrm{MB}$ strategies $\left.{ }^{10}\right)$. We propose that this account may provide a better framework to understand emotion regulation. Take the example of avoidance, which is a behavioural strategy prevalent in anxiety disorders ${ }^{11}$. The initial choice to avoid something aversive may be a MF process: it need not require a prospective model given its strong history of negative reinforcement (alleviated fear). However, once this goal has been selected, the steps to carry out avoidance may require a 'nested' MB process. An individual with a height phobia may reflexively avoid crossing bridges on their way home (MF), but may use a number of flexible, complex strategies (MB) to achieve this goal, such as taking a novel, circuitous route.

Using these nuanced classifications can provide a path forward. We propose that emotion regulation strategies should be classified on the basis of the degree to which they involve $\mathrm{MB}$ elements (for example, prospection ${ }^{12}$ ) and $\mathrm{MF}$ elements (for example, linking action and reinforcement), and the hierarchical relationships between them. This classification will enable us to predict the utility of these strategies across individuals ${ }^{12,13}$ (for example, in healthy versus clinical populations) and contexts (for example, under stress ${ }^{14}$ or cognitive load ${ }^{15}$ ).

Candace M. Raio and Peter Sokol-Hessner are at the Center for Neural Science, New York University, 4 Washington Place, Room 909, New York, New York 10003, USA.

Elizabeth V. Goldfarb, Karolina M. Lempert and Peter Sokol-Hessner are at the Department of Psychology, New York University, 6 Washington Place, Room 890 , New York, New York 10003, USA.

Correspondence to C.M.R. cmr385@nyu.edu

doi: 10.1038/nrn.2016.78 Published online 9 Jun 2016

1. Etkin, A., Büchel, C. \& Gross, J. J. The neural bases of emotion regulation. Nat. Rev. Neurosci. 16, 693-700 (2015).

2. Daw, N. D., Gershman, S. J., Seymour, B., Dayan, P. ¿ Dolan, R. J. Model-based influences on humans' choices and striatal prediction errors. Neuron 69 , 1204-1215 (2011).

3. Pavlov, I. in Conditioned Reflexes Ch. 4 (ed. Anrep, C. V.) 48-67 (Oxford Univ. Press, 1927).

4. Bouton, M. E. Context, ambiguity, and unlearning: sources of relapse after behavioral extinction. Biol. Psychiatry 52, 976-986 (2002).

5. Rescorla, R. A. \& Wagner, A. R. in Classical Conditioning II: Current Research and Theory (eds Black, A. H. \& Prokasy, W. F.) 64-99 (AppletonCentury-Crofts, 1972).

6. Gershman, S. J., Jones, C. E., Norman, K. A., Monfils, M. H. \& Niv, Y. Gradual extinction prevents the return of fear: implications for the discovery of state. Front. Behav. Neurosci. 7, 164 (2013).

7. Gershman, S. J. \& Hartley, C. Individual differences in learning predict the return of fear. Learn. Behav. 43, 243-250 (2015)

8. Gershman, S. J., Norman, K. A. \& Niv, Y. Discovering latent causes in reinforcement learning. Curr. Opin. Behav. Sci. 5, 43-50 (2015).

9. Dunsmoor, J. E., Niv, Y., Daw, N. \& Phelps, E. A Rethinking extinction. Neuron 88, 47-63 (2015)

10. Cushman, F. \& Morris, A. Habitual control of goal selection in humans. Proc. Natl Acad. Sci. USA 112 , 13817-13822 (2015).

11. Eifert, G. H. \& Forsyth, J. P. in Understanding Behavior Disorders: A Contemporary Behavioral Perspective (eds Woods, D. W. \& Kanter, J. W.) 81-116 (Context Press, 2007)

12. Doll, B. B., Duncan, K. D., Simon, D. A., Shohamy, D. $\&$ Daw, N. D. Model-based choices involve prospective neural activity. Nat. Neurosci. 18, 767-772 (2015).

13. Skatova, A., Chan, P. A. \& Daw, N. D. Extraversion differentiates between model-based and model-free strategies in a reinforcement learning task. Front. Hum Neurosci. 7, 525 (2013).

14. Otto, A. R., Raio, C. M., Chiang, A., Phelps, E. A. \& Daw, N. D. Working-memory capacity protects model-based learning from stress. Proc. Natl Acad. Sci. USA 110, 20941-20946 (2013).

15. Otto, A. R., Skatova, A., Madlon-Kay, S. \& Daw, N. D. Cognitive control predicts use of model-based reinforcement learning. J. Cogn. Neurosci. 27, 319-333 (2015)

Competing interests statement

The authors declare no competing interests. 